\title{
MISCONCEPTIONS IN PHYSICS: AN UPHILL CLIMB
}

\author{
Matteo Bozzi ${ }^{1}$, Patrizia Ghislandi ${ }^{2}$, Maurizio Zani ${ }^{1}$ \\ ${ }^{1}$ Department of Physics, Politecnico di Milano (ITALY) \\ ${ }^{2}$ Department of Psychology and Cognitive Sciences, Università degli Studi di Trento (ITALY)
}

\begin{abstract}
Constructivist science education research has pointed out that students who begin their academic career in a scientific programme may generally reveal some misconceptions on a broad spectrum of Physics topics. Our research aims at verifying if the background knowledge in classical Physics of some Politecnico di Milano learners highlights the presence of some misconceptions and their spread. Furthermore, does attending university for some months reduce these misconceptions?
\end{abstract}

Both first-year and second-year university students enrolled for engineering were involved in our study; on balance, they were 989. Data about these possible misconceptions were gathered through the use of an ad hoc test, an original Physics Concept Inventory, which was administered to all the students through the online portal Socrative and their own electronic devices, aligning with the Bring Your Own Device (BYOD) strategy. The trial consisted of 12 multiple choice quizzes; every question was characterised by four possible answers, but only one alternative out of four was correct. Therefore, the possible misconceptions investigated by means of that quiz were generally explored through the other three incorrect answers.

Actually, in accordance with the incorrect alternative selected by a student it was possible to detect their misconception related to that topic. In accord with the significant bulk of data collected one can argue that misconceptions are broadly disseminated among freshmen as well as second-year university students. On balance, freshmen often start their academic career with numerous erroneous viewpoints on Physics phenomena and attending university courses at times appear to be unable to improve significantly the students' understanding of them.

Keywords: misconceptions, Physics, study method, Physics Concept Inventory, BYOD

\section{INTRODUCTION}

Constructivist science education research has pointed out that university learners do not start with a clean slate on the physical world [1-4]. Indeed, students who begin their academic career in a scientific programme, like engineering, science, chemistry and biology, may generally reveal some erroneous viewpoints and incorrect interpretative schemas of a broad spectrum of Physics topics [510].

Students' wrong ideas and lines of reasoning on a considerable number of physical phenomena, accumulated over the years from their previous learning and experience, were christened "misconceptions" for the first time in 1972 [11] in an article that illustrated a study focused on elementary school children. Thoroughly investigated, misconceptions have assumed a more and more paramount role in Physics teaching, being classified into five different classes or categories: preconceived notions, nonscientific beliefs, conceptual misunderstanding, vernacular misconceptions and factual misconceptions $[10,12]$. From all accounts, they are essentially defined as ideas at variance with recognised views [13] or dissimilar from the ones generally acknowledged by scientists [14]. Needless to say, not only are these alternative conceptions considered as inconsistent with physicists' accepted views, but at the same time they could represent an obstacle to the process of maturation of a correct canonical students' understanding.

Born and developed into the panorama of this instructional issue, the Experimental Teaching Lab ST2 ${ }^{1}$ of Politecnico di Milano and another Italian institution, Università degli Studi di Trento, which provides pedagogic support, have developed a case study aimed at answering the following research questions:

\footnotetext{
${ }^{1}$ http://www.st2.fisi.polimi.it
} 
1. do university students enrolled for Engineering at Politecnico di Milano reveal any misconceptions in Physics, related to notable topics addressed in their academic basic Physics courses and studied at high school?

2. How widespread are these incorrect ideas?

3. Given the important role of social interaction in framing intuitive thinking, does attending university for some months reduce these misconceptions? Furthermore, is the aforementioned potential influence connected up with progress in either students' study method, or knowledge or both?

\section{METHODOLOGY}

Our research is focused on identifying and analysing some remarkable and general misconceptions shown by both first-year and second-year university students enrolled for engineering, in relation to their knowledge of Physics, with specific reference to Mechanics, Thermodynamics and Electromagnetism. These misconceptions were selected taking into account the next conditions:

a. to be pertained to some topics taught in the university Physics courses of Politecnico di Milano;

b. to be concerned in some issues that these undergraduates studied at high school.

The study target consisted in students enrolled for four different engineering branches, "Chemical Engineering", "Materials and Nanotechnology Engineering", "Mathematical Engineering" and "Physics Engineering" in the academic year 2018-2019. In respect of the purpose of our research, the undergraduates were assembled in three distinct sections named 1,2 and 3 respectively, on the basis of the Physics course they were about to attend (Experimental Physics A+B, Experimental Physics I and Experimental Physics II) and their engineering branches, as well as their year and the term in which they attended their Physics course. Table 1 synthesises the data collected with relation to the basic Physics courses involved in our study.

Table 1. Data about the basic Physics courses involved in the research.

\begin{tabular}{cccccc}
\hline Section & $\begin{array}{c}\text { Students } \\
\text { number }\end{array}$ & $\begin{array}{c}\text { Physics } \\
\text { course }^{1}\end{array}$ & $\begin{array}{c}\text { Engineering } \\
\text { study course }\end{array}$ & $\begin{array}{c}\text { Students } \\
\text { year }\end{array}$ & $\begin{array}{c}\text { Course } \\
\text { term }\end{array}$ \\
\hline 1 & 449 & Experimental Physics A+B & Chemical, \\
& & Materials and Nanotechnology & 1 & 1 \\
\hline 2 & 370 & Experimental Physics II & Mathematical, & 1 \\
\hline 3 & 170 & Experimental Physics I & Physics & 2 & 1 \\
\hline
\end{tabular}

\footnotetext{
${ }^{1}$ At Politecnico di Milano the course title can use some alphabetic letters or Roman numerals to identify the content topics. For instance, in this case $\mathrm{A}+\mathrm{B}, \mathrm{I}$ and II mean that the course regards Mechanics and Electromagnetism, Mechanics and Thermodynamics, and Electromagnetism and Optics respectively.
}

It is appropriate to point out that the students grouped in section 1 (S1) were at the start of their university career; consequently, they had never attended an academic course and their knowledge along with understanding of Physics phenomena was related to their own previous experience and instruction. Differently, even though section 3 (S3) consisted of first-year university students, like S1, these latter freshmen were at the beginning of the second academic term, hence they had already taken some university courses [15], among which Chemistry. Notwithstanding that they had not studied Physics in a previous academic course, some issues related to Thermodynamics and Electromagnetism still had been addressed in their chemistry classes. Finally, the second-year university students included in section 2 (S2) were at the beginning of their second academic year; needless to say, they had already attended a good deal of university courses [15], including Chemistry and, more importantly, Experimental Physics I which focused on Mechanics and Thermodynamics. 
To investigate the possible students' misconceptions in Physics, researchers have adopted different techniques over the year, for instance interviews [16], open-ended tests [17] and multiple-choice tests [18]. Since the number of overall students potentially involved in this study had been estimated to be massive when the research was planned - on balance they were 989 -, a multiple-choice test appeared to be an appropriate and clever option to carry out our study. Although standardised questionnaires on these subjects, statistically well validated, were undoubtedly available $[19,20]$, these trials were not designed to meet our requirements. As a consequence, the Experimental Teaching Lab ST2 of Politecnico di Milano created an authentic ad hoc multiple-choice test, identical for S1, S2 and S3, on the basis of the students' most recurrent mistakes in their Physics courses final examination, their more frequent questions during lessons or drills as well as researchers' own teaching experience and the literature on misconceptions in Physics. Università degli Studi di Trento corroborated the educational and didactic suitability of how this trial was created. This test, administered at the beginning of every university Physics course involved in our study, consisted of twelve quizzes, divided equally among Mechanics, Thermodynamics and Electromagnetism. The overall number of quizzes was set taking into account some priorities; on the one hand, the trial could not last too much, on the other hand it was essential to have an adequate number of quizzes. Every question was characterised by four possible answers, but only one alternative out of four was correct; therefore, the possible misconceptions investigated by means of that quiz were generally explored through the other three incorrect answers. Actually, in accordance with the incorrect alternative selected by a student it was possible to detect their misconception related to that topic.

The sequence of the questions and the time allotted to each one, 90 seconds, were formerly arranged. As a result, the students could not manage their time, nor the order to answer the quizzes. The trial was administered to all the students through the online portal Socrative [21] and their own electronic devices, like smartphones, tablets and laptops [22], aligning with the Bring Your Own Device (BYOD) strategy [23]. During the test, which was taken by the undergraduates during their first lecture of the course, a tutor assisted the teacher to control the whole trial: however, no interaction with the students was allowed nor suggestions to them were provided with relation to the correct answers. In table 2 investigated misconceptions, specific headings to which these aforementioned misconceptions are linked and their respective macro-areas of Physics are summarised.

Table 2. Quizzes classification based on misconception areas, specific headings and macroareas of Physics.

\begin{tabular}{|c|c|c|c|}
\hline Quiz & Misconception area & Specific heading & Macro-area \\
\hline M1 & Displacement and distance travelled & Kinematics & Mechanics \\
\hline M2 & Static friction & Force & Mechanics \\
\hline M3 & Impulse, momentum law & Linear momentum & Mechanics \\
\hline M4 & Conservation of energy, linear and angular momentum & Gravitation & Mechanics \\
\hline T1 & Phase transition and heat exchanged & Thermodynamics processes & Thermodynamics \\
\hline T2 & Relation between heat and energy of a Thermodynamics system & Heat & Thermodynamics \\
\hline T3 & Quasi-static and no quasi-static adiabatic process & Thermodynamics processes & Thermodynamics \\
\hline T4 & Efficiency of a heat engine and comparison with Carnot engine & Heat engine & Thermodynamics \\
\hline E1 & Source of an electric field & Electric field & Electromagnetism \\
\hline E2 & Electrical induction & Conductors & Electromagnetism \\
\hline E3 & Electric field in the presence of a dielectric & Dielectrics & Electromagnetism \\
\hline E4 & Equilibrium of a current loop lying in a magnetic field & Magnetostatics & Electromagnetism \\
\hline
\end{tabular}




\section{RESULTS}

The first step of our analysis consisted in evaluating the overall results of the test, comparing and contrasting the students' groups of the three sections involved in the research, in order to gauge how widespread possible misconceptions in Physics were. Moreover, the level of difficulty of every single quiz was determined according to the number of correct answers given to it by the students: it was classified as easy (E) if the right answers were more than 70\%, difficult (D) if they were less than 35\%, regular $(R)$ otherwise $[24,25]$. Tables 3 shows these data related to each quiz and every students' section engaged in the study.

Table 3. Percentage of correct answers and relative difficulty related to each quiz and students' section involved in the research.

\begin{tabular}{|c|c|c|c|}
\hline & \multicolumn{3}{|c|}{ Section } \\
\hline Quiz & 1 & 2 & 3 \\
\hline M1 & $15,6 \%-D$ & $15,7 \%-D$ & $10,6 \%-D$ \\
\hline M2 & $25,6 \%-D$ & $11,4 \%-D$ & $20,0 \%-D$ \\
\hline M3 & $32,1 \%-D$ & $79,5 \%-E$ & $38,2 \%-R$ \\
\hline M4 & $13,8 \%-D$ & $4,6 \%-D$ & $10,0 \%-D$ \\
\hline T1 & $54,1 \%-\mathrm{R}$ & $79,7 \%-E$ & $76,5 \%-E$ \\
\hline T2 & $52,6 \%-R$ & $68,9 \%-R$ & $72,9 \%-E$ \\
\hline T3 & $16,9 \%-D$ & $54,3 \%-R$ & $20,0 \%-D$ \\
\hline T4 & $54,3 \%-R$ & $47,6 \%-\mathrm{R}$ & $54,7 \%-\mathrm{R}$ \\
\hline E1 & $38,3 \%-\mathrm{R}$ & $53,2 \%-R$ & $58,8 \%-R$ \\
\hline E2 & $8,5 \%$ - D & $21,1 \%-D$ & $15,3 \%-D$ \\
\hline E3 & $48,1 \%-\mathrm{R}$ & $46,0 \%-R$ & $54,1 \%-R$ \\
\hline E4 & $13,1 \%-D$ & $8,1 \%-D$ & $5,9 \%-D$ \\
\hline
\end{tabular}

The first-year university students (S1 and S3) who selected the correct alternative were generally few; as regards freshmen attending their Physics course in the first term (S1), the rate of correct answers was permanently inferior to $60 \%$, no question could be classified as easy and seven out of twelve were categorised as difficult. Comparable considerations should apply to freshmen who took their Physics course in the second term (S3): the percentage of correct answers was superior to $60 \%$ only twice and the difficult questions were one out of two. Moreover, it ought to be emphasised that the findings concerning the second-year university students (S2) were not blatantly better and in some cases the rate of their success was undoubtedly lower: the percentage of correct answers was higher than $60 \%$ just three times out of twelve and only two quizzes might be classified as easy.

In short, these data highlight that these misconceptions are broadly widespread among the students of all the three sections and are generally related to every macro-area involved.

A second phase of the analysis process consisted in examining the outcomes of the trial with specific reference to each incorrect answer to every quiz, comparing and contrasting the students' groups of the three sections involved in the study. Furthermore, the three macro-areas were analysed separately.

Table 4 and figure 1 summarise the data related to each quiz in the macro-area named Mechanics expressed by every section. 
Table 4. Results related to answers A-D to each quiz of the Mechanics macro-area given by the students' sections involved in the research. The correct alternative is highlighted in bold.

\begin{tabular}{|c|c|c|c|c|c|c|}
\hline Quiz & Section & A & B & C & D & No answer \\
\hline \multirow{3}{*}{ M1 } & 1 & $51,9 \%$ & $15,6 \%$ & $10,2 \%$ & $18,7 \%$ & $3,6 \%$ \\
\hline & 2 & $54,3 \%$ & $15,7 \%$ & $8,7 \%$ & $19,5 \%$ & $1,9 \%$ \\
\hline & 3 & $60,6 \%$ & $10,6 \%$ & $11,2 \%$ & $15,9 \%$ & $1,8 \%$ \\
\hline \multirow{3}{*}{ M2 } & 1 & $28,5 \%$ & $30,5 \%$ & $12,3 \%$ & $25,6 \%$ & $3,1 \%$ \\
\hline & 2 & $44,3 \%$ & $38,4 \%$ & $3,0 \%$ & $11,4 \%$ & $3,0 \%$ \\
\hline & 3 & $34,7 \%$ & $32,9 \%$ & $11,2 \%$ & $20,0 \%$ & $1,2 \%$ \\
\hline \multirow{3}{*}{ M3 } & 1 & $44,5 \%$ & $32,1 \%$ & $11,8 \%$ & $7,8 \%$ & $3,8 \%$ \\
\hline & 2 & $11,1 \%$ & $79,5 \%$ & $3,8 \%$ & $3,5 \%$ & $2,2 \%$ \\
\hline & 3 & $44,1 \%$ & $38,2 \%$ & $8,8 \%$ & $6,5 \%$ & $2,4 \%$ \\
\hline \multirow{3}{*}{ M4 } & 1 & $13,8 \%$ & $37,6 \%$ & $24,7 \%$ & $22,1 \%$ & $1,8 \%$ \\
\hline & 2 & $4,6 \%$ & $76,5 \%$ & $3,5 \%$ & $13,8 \%$ & $1,6 \%$ \\
\hline & 3 & $10,0 \%$ & $48,8 \%$ & $12,9 \%$ & $22,4 \%$ & $5,9 \%$ \\
\hline
\end{tabular}

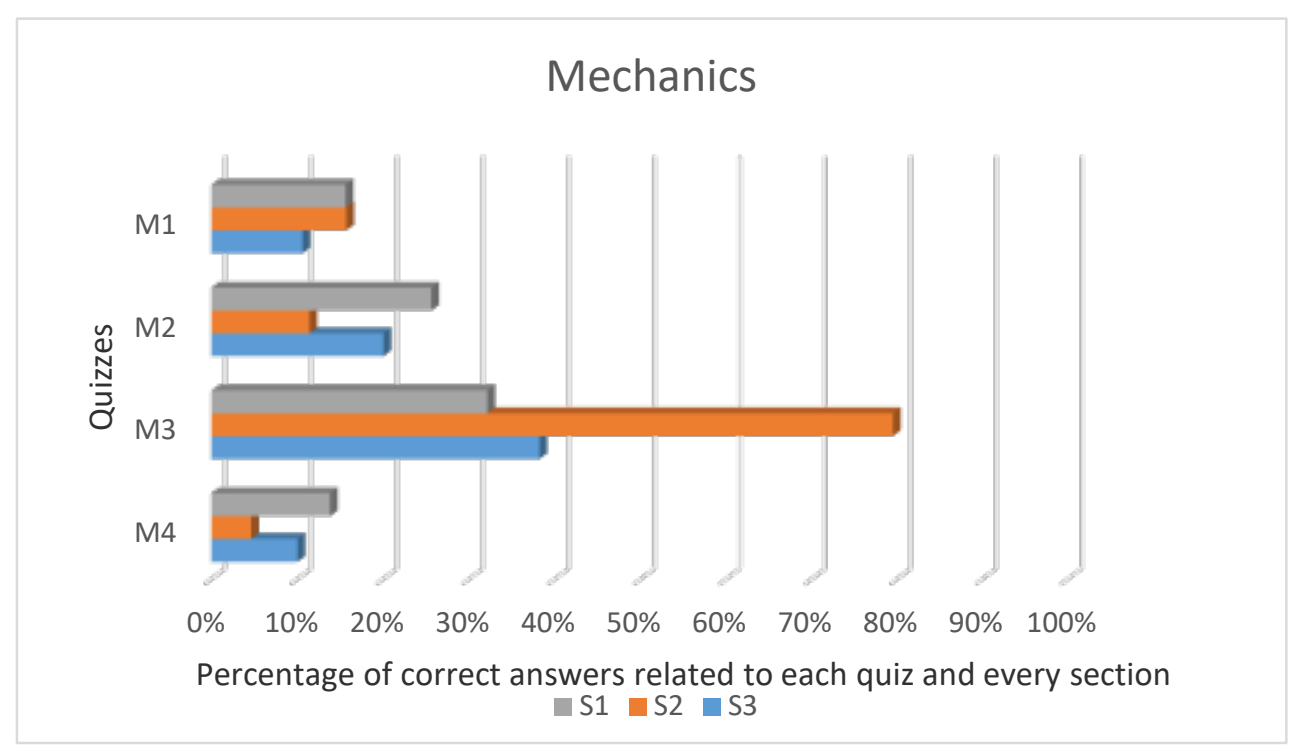

Figure 1. Results related to correct answers to each quiz of the Mechanics macro-area given by the students' sections involved in the research.

The table and the figure highlight the fact that the results show no significant difference among freshmen (S1 and S3) and between the just mentioned first-year university students and the secondyear university students (S2), with the exception of quiz M3 where the latter appear to overcome largely the misconceptions related to that specific topic. In quizzes M1 and M3 the incorrect answers of all the students engaged in the research predominantly focus on one of the possible alternatives (A), so that one can argue that there is only one predominant misconception related to those Physics subject matters. With reference to the Physics topics addressed in quizzes M2 and M4 the issue is more complex owing to at least two incorrect answers overwhelmingly chosen by students, namely $A$ and $B$ for quiz $M 2$ and $B$ and $D$ for quiz M4; it implies that there are multiple misconceptions 
influencing their knowledge on those Physics themes. However, one should emphasise that most of S2 students, who had attended a Physics course where these Mechanics topics had already been studied, opted for the only incorrect alternative B in quiz M4. It could be asserted that attending that Physics course may have resulted in the students' strengthening of one of the possible misconceptions rather than getting through all of them.

Table 5 and figure 2 summarise data related to every question allotted in the macro-area Thermodynamics expressed by each section.

Table 5. Findings related to answers A-D to each quiz of the Thermodynamics macro-area given by the students' sections involved in the research. The correct alternative is highlighted in bold.

\begin{tabular}{|c|c|c|c|c|c|c|}
\hline Quiz & Section & A & B & C & D & No answer \\
\hline \multirow{3}{*}{ T1 } & 1 & $2,2 \%$ & $11,8 \%$ & $54,1 \%$ & $29,0 \%$ & $3,1 \%$ \\
\hline & 2 & $4,6 \%$ & $6,5 \%$ & $79,7 \%$ & $7,3 \%$ & $1,9 \%$ \\
\hline & 3 & $4,1 \%$ & $4,7 \%$ & $76,5 \%$ & $10,6 \%$ & $4,1 \%$ \\
\hline \multirow{3}{*}{ T2 } & 1 & $27,8 \%$ & $52,6 \%$ & $16,7 \%$ & $0,67 \%$ & $2,2 \%$ \\
\hline & 2 & $17,6 \%$ & $68,9 \%$ & $11,9 \%$ & $0,27 \%$ & $1,4 \%$ \\
\hline & 3 & $17,7 \%$ & $72,9 \%$ & $7,7 \%$ & $0,59 \%$ & $1,2 \%$ \\
\hline \multirow{3}{*}{ T3 } & 1 & $16,9 \%$ & $25,2 \%$ & $15,4 \%$ & $34,3 \%$ & $8,2 \%$ \\
\hline & 2 & $54,3 \%$ & $6,2 \%$ & $18,1 \%$ & $18,4 \%$ & $3,0 \%$ \\
\hline & 3 & $20,0 \%$ & $16,5 \%$ & $11,8 \%$ & $42,9 \%$ & $8,8 \%$ \\
\hline \multirow{3}{*}{ T4 } & 1 & $24,5 \%$ & $14,5 \%$ & $54,3 \%$ & $1,6 \%$ & $5,1 \%$ \\
\hline & 2 & $32,4 \%$ & $12,2 \%$ & $47,6 \%$ & $1,35 \%$ & $6,5 \%$ \\
\hline & 3 & $25,9 \%$ & $6,5 \%$ & $54,7 \%$ & $2,94 \%$ & $10,0 \%$ \\
\hline
\end{tabular}

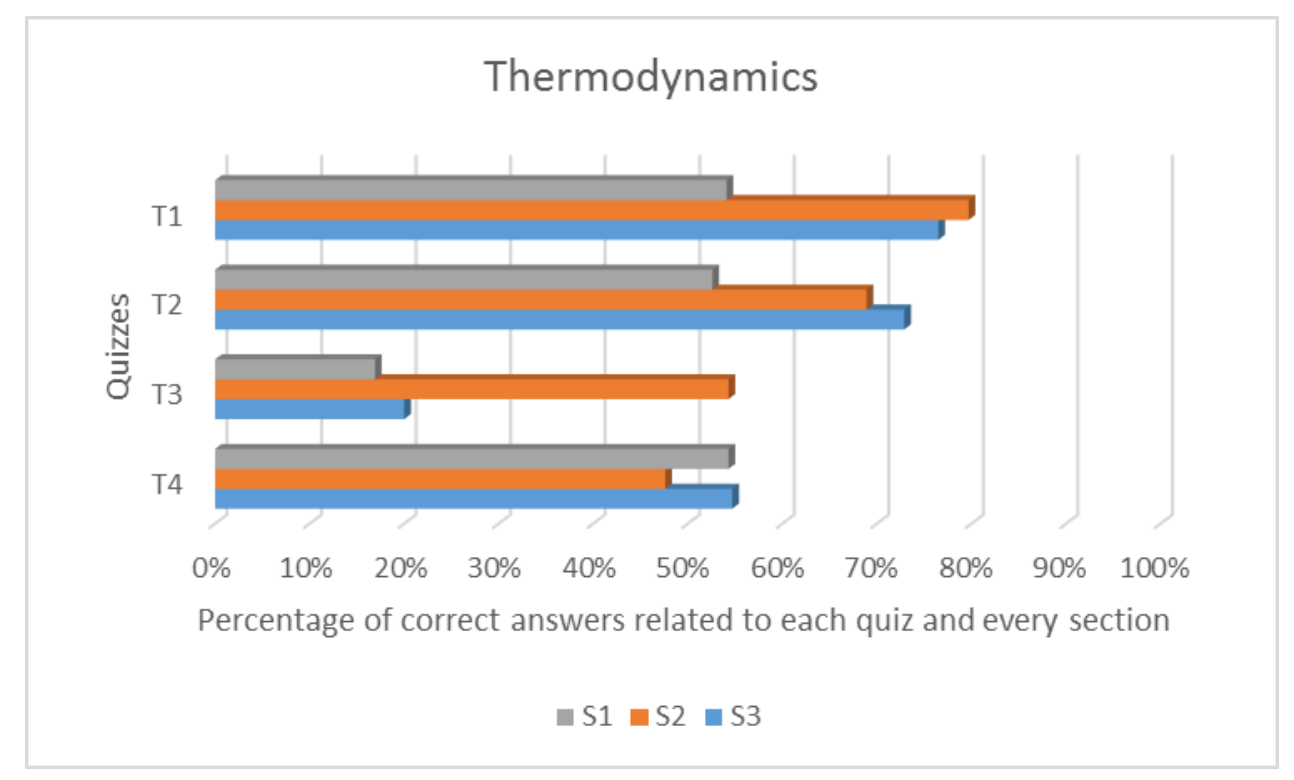

Figure 2. Results related to correct answers to each quiz of the Thermodynamics macro-area given by the students' sections involved in the research. 
The table and the figure show that S1 freshmen, who had never attended any university course, revealed erroneous viewpoints on all the quizzes, blatantly in question T3 where two incorrect answers were particularly taken into account by students. Considering S3 freshmen, their outcomes were significantly different in comparison with S1 in quizzes T1 and T2, where misconceptions do not appear to be broadly widespread. Indeed, those issues had been dealt with in the Chemistry course that they had just taken. On the contrary, the results of these two groups were homogeneous in questions T3 and T4, based on topics not addressed before, even though S3 freshmen significantly selected only one of the possible incorrect answers (D) in quiz T3. In conclusion, S2 students, who had studied Thermodynamics both in a Physics and a Chemistry course during their first academic year, highlighted achievements similar to S3 freshmen, with the exception of quiz T3, where wrong answers were characterised by a lower, though not negligible, rate.

Table 6 and figure 3 show data related to each quiz in the macro-area named Electromagnetism expressed by every section.

Table 6. Outcomes related to answers A-D to each quiz of the Electromagnetism macro-area given by the students' sections involved in the research. The correct alternative is highlighted in bold.

\begin{tabular}{|c|c|c|c|c|c|c|}
\hline Quiz & Section & A & B & C & D & No answer \\
\hline \multirow{3}{*}{ E1 } & 1 & $18,7 \%$ & $9,1 \%$ & $38,3 \%$ & $29,0 \%$ & $4,9 \%$ \\
\hline & 2 & $14,3 \%$ & $8,1 \%$ & $53,2 \%$ & $15,1 \%$ & $9,2 \%$ \\
\hline & 3 & $8,8 \%$ & $4,7 \%$ & $58,8 \%$ & $24,7 \%$ & $2,9 \%$ \\
\hline \multirow{3}{*}{ E2 } & 1 & $25,8 \%$ & $38,5 \%$ & $24,9 \%$ & $8,5 \%$ & $2,2 \%$ \\
\hline & 2 & $20,3 \%$ & $33,0 \%$ & $17,0 \%$ & $21,1 \%$ & $8,7 \%$ \\
\hline & 3 & $22,4 \%$ & $40,0 \%$ & $20,6 \%$ & $15,3 \%$ & $1,8 \%$ \\
\hline \multirow{3}{*}{ E3 } & 1 & $20,9 \%$ & $12,5 \%$ & $11,6 \%$ & $48,1 \%$ & $6,9 \%$ \\
\hline & 2 & $25,4 \%$ & $7,8 \%$ & $10,8 \%$ & $46,0 \%$ & $10,0 \%$ \\
\hline & 3 & $22,4 \%$ & $9,4 \%$ & $10,6 \%$ & $54,1 \%$ & $3,5 \%$ \\
\hline \multirow{3}{*}{ E4 } & 1 & $27,6 \%$ & $13,1 \%$ & $14,7 \%$ & $41,0 \%$ & $3,6 \%$ \\
\hline & 2 & $30,3 \%$ & $8,1 \%$ & $15,7 \%$ & $36,8 \%$ & $9,2 \%$ \\
\hline & 3 & $35,9 \%$ & $5,9 \%$ & $11,8 \%$ & $44,7 \%$ & $1,8 \%$ \\
\hline
\end{tabular}




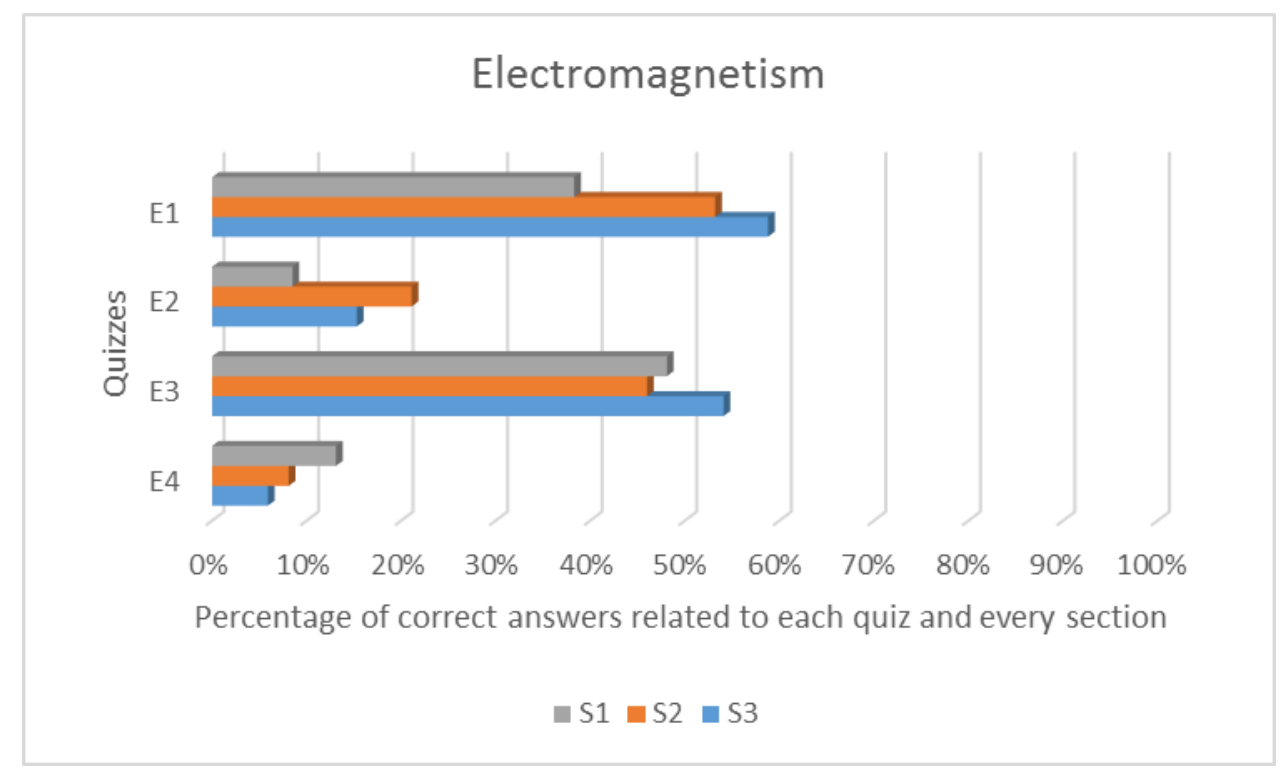

Figure 3. Results related to correct answers to each quiz of the Electromagnetism macro-area given by the students' sections involved in the research.

The table and the figure highlight the impressive diffusion of misconceptions with relation to quizzes E2 and E4 among all the students engaged in the study. At least two incorrect alternatives are characterised by a high score. Taking into account questions E1 and E3, misconceptions appear to play an appreciable role; in fact, notwithstanding that the rate of correct answers is higher than in the quizzes previously mentioned it is still lower than $60 \%$. It is worth to emphasise the unusual number of unfilled answers which characterised S2.

On balance, considering that S2 and S3 students had already attended a university Chemistry course, where they had studied some preliminary issues about Electrostatics, all the three sections attained substantially equivalent outcomes in Electromagnetism. As a consequence, although to attend university for six months (S3) or one year (S2) probably contributes to developing learners' general and integral processes of constant growth, it could be argued that this attendance does not appear to change some incorrect frames of mind that students employ to interpret the physical reality. Comparing S1 and S3 results in Mechanics seems to confirm this claim.

In other words, with relation to the third research question one might conclude that the study of some Physics topics in some university courses by undergraduates enhances slightly their Physics knowledge. On the contrary, attending university for some months does not appear to be effective in fostering significant progress in students' study method.

\section{CONCLUSIONS}

Our research corroborates the awareness that both Politecnico di Milano freshmen and second-year students frequently highlight some incorrect ideas and erroneous interpretative schemas on many Physics phenomena: only $35.7 \%$ of the answers in the multiple-choice test administered to the learners was correct. Indeed, to the present day to attend a Physics course as well as to succeed in its final examination rarely allows students to overcome many of these erroneous interpretative schemas; on the contrary, an initial misconception may be more frequently replaced by a different one related to the same topic. In this respect we are planning to redesign some High School and academic Physics lectures where possible solutions, chosen on an empirical base, will be tested and their effectiveness checked.

It is worth to emphasise that enhancing the scientific background of the prospective freshmen helps to reduce their future learning difficulties as well as to increase students' self-efficacy and self-esteem; accordingly, it is likely to decrease the rate of failure at academic final exams and the number of students who could potentially drop out [26] or need extra time to conclude their academic career. These phenomena have a thorough impact on the issue of sustainability owing to the waste of 
economic and personal resources and time [27]. Actually, Politecnico di Milano is strongly committed to improving the sustainability of its educational activity and a new project named ProPre ("Profiling and Predicting PoliMi students' performance at Bachelor and Master courses, using Advanced Statistical Methods and Machine Learning Techniques"), which has been funded by the Italian Government and the university itself, will further strengthen these efforts. Our study moves in this direction.

\section{ACKNOWLEDGEMENTS}

We thank Claudia Andreani for editing this article.

\section{REFERENCES}

[1] J. Piaget, B. Inhelder, "Le développement des quantités Physiques chez l'enfant: conservation et atomisme (3e éd)". Neuchatel-Paris: Delachaux \& Niestlé, 1968.

[2] A. A. diSessa, "Toward an Epistemology of Physics", Cognit. Instr., vol. 10, pp. 105-225, 1993.

[3] A. Elby, "Helping physics students learn how to learn", Am. J. Phys., vol. 69, pp. S54-S64, 2001.

[4] D. Hammer, "Student resources for learning introductory physics", Am. J. Phys., vol. 68, pp. S52-S59, 2000.

[5] J. Warren, "Circular motion", Physics Education, vol. 6, pp.74-77, 1971.

[6] H. Helm, "Misconceptions in physics amongst South African students", Phys. Educ., vol. 15, pp. 92-105, 1980.

[7] A. Brown, S. David, J. Clement, "Misconceptions concerning Newton's law of action reaction", Proceedings of the Second International Seminar Misconceptions and Educational Strategies in Science and Mathematics, Ithaca, NY (USA): Cornell University, vol. 3, pp. 39-53, 1987.

[8] L. C. McDermott, P. S. Shaffer, "Research as a guide for curriculum development: An example from introductory electricity. Part I: Investigation of student understanding", Am. J. Phys., vol. 60, pp. 994-1003, 1992.

[9] M. Planinic et al., "Exploring Alternative Conceptions from Newtonian Dynamics and Simple DC Circuits: Links between Item Difficulty and Item Confidence", JRST, vol. 43, pp. 150-171, 2006.

[10] M. Bozzi, P. Ghislandi, M. Zani et al., "Highlight misconceptions in Physics: a T.I.M.E. project", Proceedings of INTED2019, pp. 2520-2525, Valencia, Spain: IATED, 2019.

[11] R. L. Doran, "Misconceptions of Selected Science Concepts", Journal of Research in Science Teaching, vol. 9, pp. 127-137, 1972.

[12] Committee on Undergraduate Science Education, "Misconceptions as Barriers to understanding Science" in Science Teaching Reconsidered: A Handbook, pp. 27-32, Washington, D. C.: National Academies Press, 1997.

[13] K. M. Fisher, J. I. Lipson, "Ten rules of thumb: Information processing interpretations of error research in learning", Proceedings of the International Seminar on misconceptions in Science and Mathematics, Ithaca, NY (USA): Cornell University, pp. 150-152, 1983.

[14] A. L. Odom, L. H. Barrow, "Development and application of a two-tier diagnostic test measuring college biology students' understanding of diffusion and osmosis after a course of instruction", Journal of Research in Science Teaching, vol. 32, pp. 45-61, 1995.

[15] Manifesto degli studi. Politecnico di Milano. Available online: https://www4.ceda.polimi.it/manifesti/manifesti/controller/ManifestoPublic.do (accessed on 20/10/2019)

[16] J. Park, S. Han, 'Using deductive reasoning to promote change of students' conceptions about force and motion", International Journal of Science Education, vol. 24, no. 6, pp. 593-609, 2010.

[17] P. Colin, F. Chauvet, L. Viennot, "Reading images in optics: students' difficulties and teachers' views", International Journal of Science Education, vol. 24, no. 3, pp. 313-332, 2002. 
[18] T. Martìn-Blas, L. Seidel, A. Serrano-Fernàndez, "Enhancing Force Concept Inventory diagnostics to identify dominant misconceptions in first-year engineering physics", European Journal of Engineering Education, vol. 35, 6, pp. 597-606, 2010.

[19] D. Hestenes et al., "Force Concept Inventory", Phys. Teach., vol. 30, pp. 141-158, 1992.

[20] G. W. Fulmer et al., "Applying a force and motion learning progression over an extended time span using the Force Concept Inventory", Int. J. Sci. Educ., vol. 36, pp. 2918-2936, 2014.

[21] M. F. Tretinjak, A. Bednjanec, M. Tretinjak, "Interactive Teaching with Socrative", Proceedings of MIPRO 2015, pp. 848-851, Opatija, Croatia, 2015.

[22] D. M. Coca, J. Slisko, "Software Socrative and smartphones as tools for implementation of basic processes of active physics learning in classroom: An initial feasibility study with prospective teachers", EJPE, vol. 4, pp. 17-24, 2013.

[23] R. Afreen, "Bring Your Own Device (BYOD) in Higher Education: Opportunities and Challenges", IJETTCS, vol. 3, pp. 233-236, 2014.

[24] C. H. Crouch, E. Mazur, "Peer instruction: Ten years of experience and results", Am. J. Phys., vol. 69, pp. 970-977, 2001.

[25] M. Bozzi, J. Raffaghelli, M. Zani, "Peer learning for large size Physics lectures in higher education: yes, we can", Proceedings of ICERI2018, pp. 8739-8747, Seville, Spain: IATED, 2018.

[26] M. T. H. Chi, "Commonsense Conceptions of Emergent Processes: Why Some Misconceptions Are Robust", The Journal of the Learning Sciences, vol. 14, pp. 161-199, 2005.

[27] J. Oldfield et al., "Psychological and demographic predictors of undergraduate nonattendance at university lectures and seminars", Journal of Further and Higher Education, vol. 42, pp. 509-523, 2018. 